\section{THE CAUSES OF COLITIS, WITH SPECIAL REFERENCE TO ITS SURGICAL TREATMENT. ${ }^{1}$}

WITH AN ACCOUNT OF 36 CASES.

BY J. P. LOCKHART MUMMERY, M.B. CANTAB., F.R.C.S. ENG.,

HONORARY SURGEON, KING EDWARD VII. HOSPITAL FOR OFFICERS; ASSISTANT SURGEON TO ST. MARK'S HOSPITAL FOR DISEASES OF THE RECTUM AND TO THE NORTH-EASTERN HOSPITAL FOR CHILDREN.

A GREAT deal of attention has been paid within recent years to the subject of colitis, more especially, perhaps, on the continent, and in $1905 \mathrm{Dr}$. W. Hale White brought the subject prominently before the profession in this country by the publication of his study of 60 cases. Although colitis is generally looked upon as belonging to the province of the physician rather than that of the surgeon, many cases of colitis come under the care of the surgeon.

Colitis has been described under many different names by different authors. Such names as colica mucosa, tubular diarrhoea, membranous colitis, and muco-membranous entero-colitis are only a few of the names which have been used to describe what is without doubt the same condition. My cases do not differ in symptomatology in any important particular from those recorded by other writers under one or other of these different names. The essential symptom in all cases is the presence of excess of mucus in the stools in the form of casts (complete or broken up), shreds, or large glairy masses. In addition bleeding, intestinal sand, diarrhoea, and acute attacks of abdominal pain are common accompanying symptoms.

From the study of my own cases I have been driven to the conclusion that colica mucosa, by whatever name it is called, has no claim to be described as a disease any more than has diarrhœe or hæmatemesis, but that it is a symptom or condition which may result from a number of different diseases of the colon of widely different characters. This is not, I think, a new hypothesis, as although the point has not been specially emphasised before it is the logical conclusion to be drawn from such collections of cases as those of Dr. Hale White and some of the German writers on the subject. shall attempt to show that in the great majority of cases a lesion or lesions can be demonstrated which it is only reasonable to suppose is the cause of the condition.

Until a few years ago our only means of knowing the condition of the colon in cases of colitis was by a study of the symptoms and an examination of the abdomen and of the discharges, aided sometimes by what was seen of the outside of the bowel when an operation was performed or of its interior when the patient had died from some other cause when suffering from colitis. Within the last four or five years, however, the technique of examining the lower bowel by means of instruments has so much improved that we have been enabled to examine accurately the interior of the lower bowel in all those cases where symptoms of colitis were present, and in this way much has been learned as to the causes of colitis which was hitherto unknown.

The most important advance in our knowledge of these cases has been due to the invention of the electric sigmoidoscope. This instrument enables us to see the condition of the mucosa in the rectum and sigmoid flexure and to compare the condition found in health with that found when symptoms of colitis are present. It has rendered untenable the theory of Professor Nothnagel that colitis is a neurosis unaccompanied by local lesions of the bowel, for in quite a large proportion of the cases definite local lesions can be found to explain the symptoms.

A point of no little importance which a systematic sigmoidoscopic examination of the bowel in cases of colitis has brought out is that the causes for the condition are in many cases confined to the lower part of the sigmoid flexure and rectum. This fact was pointed out by Tuttle and Kelly and I have been able repeatedly to verify the truth of their observations. It is, after all, not surprising that this should be so as the portion of the large intestine which is most subjected to abnormal conditions is the sigmoid flexure and rectum; it is here that stagnation and pressure occur in

1 A paper read before the Royal Medical and Chirurgical Society on constipation and there can be no doubt that constipation is by far the commonest antecedent of colitis ; it is here that. any irritant material, be it solid or liquid, will collect and tend to stagnate if present in the large bowel ; and it is here that the greatest amount of traumatism from the passage of hardened fæcal masses will be felt. In fact, those portions of the large bowel which are most liable to injurious influences are the two dependent ends, the cæcnm and the sigmoid flexure, and it is here that disease when it attacks the large intestine is most frequently found. We should therefore expect that the chief lesion would be situated either in the cæcum or in the sigmoid flexure and, as I shall attempt to show, this is the case.

Symptoms. - Diarrboea was a prominent feature in a majority of the cases and in some it was most severe. In Cases 16, 20, and 23 there were at one time as many as from. 20 to 25 stools per diem. The diarrhœa was usually the symptom which first caused the patient to seek medical advice. In almost all the cases there was an antecedent history of constipation quite irrespectively of whether an inflammatory lesion or a new growth was found at the examination.

Bleeding occurred in 22 out of the 36 cases. In most of the cases in which there was bleeding this could easily be accounted for by the fact that definite ulceration was present in the bowel. In some, however, no definite cause for the bleeding was discovered. In two of the cases of new growth, Cases 18 and 19, there was no bleeding at all. This shows, I think, that bleeding cannot be considered characteristic of new growth.

Mucus was present in the stools in all the cases in sufficient quantities to draw the patient's attention to it. In some cases very large quantities of mucus were passed so that the stools consisted sometimes of little else. The mucus was in some cases thin and almost fluid, in others thick and gelatinous or in shreds and strings. Large mucous casts were present in the stools in eight of the cases, and I would especially draw attention to the fact that casts were seen in two of the cases, Cases 3 and 21, which were due to malignant disease of the bowel. These two cases are, I think, worth recording in detail.

Case 3 was that of a woman who for five years had suffered from acute attacks of pain in the abdomen followed by the passing of large mucous casts per anum. There had been some bleeding at times during the last 12 months. On examining her I found that she had a large growth in the sigmoid flexure. The growth was quite inoperable and she died a few months after I saw her.

Case 21 was that of a man who had only had symptoms of bowel trouble for six months. On several occasions he had passed mucous casts several inches in length. On examining him I found a large malignant growth in the sigmoid flexure and a mass of enlarged glands in the meso. rectum. No operation was possible in his case.

Loss of weight was quite as common in the non-malignant as in the malignant cases. It was most marked in those cases associated with severe diarrhœea.

Intestinal sand was seen in the stools in two cases (Cases 2 and 20). In both it was a fine yellowish-red sand when passed but it became black if left for some days. The sand was analysed in both cases and was found to consist of carbonates and phosphate of calcium and ammonium but no cholesterin. It is an interesting fact that in Case 2 the patient also occasionally passed uric acid gravel in the urine. In both cases very severe attacks of abdominal colic were associated with the appearance of the sand.

The interesting condition of enterospasm was present in one of my cases (Case 20). Enterospasm is a localised contraction of the bowel, often resulting in a temporary intussusception. It most usually occurs in the descending colon or sigmoid flexure and is accompanied by extreme pain localised to that portion of the abdomen in which the contracted portion of bowel lies. The spasm may involve a large length of bowel according to some authorities, but in those cases in which I have met with it only an inch or two of bowel has been contracted. In my patient there were attacks of extreme abdominal pain and a hard tumour about two inches in length could be felt in the sigmoid flexure. I examined the patient very carefully, as I suspected that her colitis might arise from a growth in the sigmoid flexure. She had constant diarrhoa with stools consisting of blood and mucus. The tumour might have easily been mistaken for a growth, but I found that it always disappeared when the pain ceased and was always present when a fresh attack 
of pain occarred. The contracted portion of bowel always occupied the same position and I came to the conclusion that the spasm resulted from an ulcer in that portion of the bowel, as there was tenderness over that spot when the contraction was not present. The patient recovered as the result of a careful dieting and irrigation.

My colleague at St. Mark's Hospital, Mr. F. Swinford Edwards, had a most interesting case of the same sort. He opened the abdomen to explore for a tumour in the sigmoid flexure and on drawing out the bowel found a contracted band about two inches in length resembling a local intussusception. While he was watching it disappeared and again reappeared. Enterospasm appears to result from some local irritation within the bowel. The irritation may result from an ulcer within the bowel or from a hardened fæcal mass or concretion.

The examination of the patient.-A patient suffering from colitis cannot, as a rule, be properly examined in the consulting-room or out-patient department of a hospital. The sigmoid flexure is normally full of fæcal material and special means are necessary to empty it. The patient should be confined to bed and the rectum and sigmoid flexure thoroughly emptied by aperients and enemata administered by a skilled nurse. The examination with the sigmoidoscope is not painfal and, as a rule, causes but little discomfort, so that an anæsthetic is not required in most cases. The greater part of the sigmoid flexure can be readily examined and the condition of the mucosa observed. The sigmoidoscope, like the cystoscope or laryngoscope, requires practice before it can be properly used, but in skilled bands it is perfectly safe and the view of the parts obtained with it is all that could be desired. The abdomen should, of course, be examined, especially the appendix area and the left flank. The stools should also be examined and in some cases a microscopical or bacteriological examination made of the abnormal constituents.

Cases of colitis in which lesions are present within the bowel.-I shall attempt to show that some lesion either within the bowel or external to it, but involving it, can be demonstrated in the majority of cases of colitis and that the recent improved methods of examining the bowel have proved that what is generally called colitis is a symptom of some local lesion of the bowel. I do not wish to assert that whenever we find an abnormal condition of the mucosa we are necessarily at once to look upon it as the cause of the colitis. I do not think that the gravity of the lesion in some of the cases is sufficient to justify this view, and it may be that the appearances are but secondary to some more serious lesion at a higher level. That this may occur is evidenced by the following case which I saw in consultation with Mr. A. W. Mayo Robson. The patient was an elderly gentleman who for many months had been passing large quantities of blood and mucus in his stools. An examination with the sigmoidoscope was made and revealed the presence of numerous small follicular ulcers scattered about on the mucous membrane of the rectum and sigmoid flexure. There seemed to be sufficient ulceration present to account for his symptoms and he was treated by irrigation and injections upon this assumption. After a few weeks, however, although the ulcers had healed, he was still passing blood and mucus in considerable quantities. He was again examined with the sigmoidoscope and on this occasion a cancerous ulcer was detected very bigh up in the sigmoid flexure. This was subsequently excised by Mr. Mayo Robson. This case shows the importance of a thorough and careful examination and that the presence of definite ulceration in the bowel does not exclude the possibility of a more serious lesion at a higher level.

It will be observed by reference to the table of cases that in the majority of cases of colitis the lesions discovered on examination were of a chronic inflammatory nature. This is opposed to the views expressed by Nothnagel who believed colitis to be in most cases a neurosis.

In my series of cases a definite inflammatory condition of the mucosa was found in 24 out of the total of 36 cases, or in 66 per cent. The appearance of the inflammatory condition found varied very considerably in the different cases and varied sometimes in the same case at different times. In all the cases the inflammation was of a chronic character and appeared to involve only the mucous membrane or submucous cellular tissue.

The most usual appearance was for the mucous membrane to be injected and bright red ; the normal mucous membrane is of a pale pink colour. The surface was dull, having lost its normal glistening appearance, and often granular. This granular condition closely resembles that seen in granular pharyngitis. In many of the cases the mucous membrane looked as if its surface had been rubbed off with sand paper. It bled readily if touched with the instrument and bleeding spots could often be seen apart from this. Scattered about on the mucous membrane there were irregular patches of yellowish white adherent mucus which often gave a very characteristic appearance to the bowel. Occasionally a very extensive area of bowel was affected, and in some cases the whole of the visible portion of the sigmoid flexure was involved. The inflammation was always most marked at the flexure and on the valves of Houston. In some of the cases, more especially when an examination was made during acute attacks, the condition resembled an extensive superficial ulceration of the mucosa.

In addition to the granular condition of the racous membrane there was definite ulceration in seven of the cases. The ulcers were irregular in outline, quite shallow, and with a granular base. These ulcers were often very numerons and sometimes extended throughout the entire sigmoid flexure and rectum. To turn to the cases in which ulceration is found. The degree of alceration which may be found in a case with symptoms of colitis varieg very considerably. Thus in some cases I have seen a few shallow alcers at irregular intervals, situated chiefly in the rectum or in the lower portion only of the sigmoid flexure. In other cases these ulcers were so numerous as to cover most of the surface of the rectum and sigmoid flexure, the normal mucosa standing out in contrast to the background of ulceration. Even in such cases, however, the ulceration may be so shallow as to involve the superficial layers only of the mucosa and it is important to distinguish between this variety and that in which the ulcers are numerous and deep. The latter is a much more serious condition; the ridges of healthy mucous membrane between the ulcerated areas here appear like polypi, so prominently do they appear to stand up from the surrounding ulcerated areas.

As a rule, ulceration is found in those cases of colitis where severe symptoms exist, but this is not always the case and I have seen well-marked ulceration in cases where the symptoms were mild and no ulceration in cases where the symptoms would lead one to suppose that severe ulceration was present. The ulcers as a rule in the more chronic cases are found at those points where local irritation or traumatism is likely to occur-thus, at the recto-sigmoidal junction, in the bottom of the sigmoid couches, and on the surfaces of the rectal valves. These shallow ulcers must be distinguished from the ulceration found in cases of acute ulcerative colitis or dysentery. In the latter conditions the whole thickness of the mucosa is destroyed and if healing occurs there is of necessity considerable scarring.

In one case I had the opportunity of examining a patient who had recovered from a severe attack of dysentery and the white stellate-looking scars left by the healed ulcers could be seen all over the rectum and sigmoid flexure. In the shallow form of ulceration, however, which I have seen associated with chronic colitis the ulcers readily heal with suitable local treatment and no scarring results. The symptoms during an acute attack may, however, be very severe, and it is important to distinguish this form of ulceration from the deeper and more serious ulceration, as the prognosis, while good in the former, is not so favourable in the latter.

CASE 36 is of particular interest. The patient was a man, aged 41 years, under the care of Dr. G. Baynton Forge. He had always been a healthy man and, with the exception of one or two slight attacks of diarrhcea, had had no serious illness until recently. In July, 1906, after a period of overwork he was suddenly tak $n$ ill with severe diarrh œea. This got worse and he began to pass blood and mucus in considerable quantities. In spite of rest and treatment there was no improvement in his condition. He was for some weeks under treatment in Maidstone Hospital but withont much benefit. When I first saw him in February of this year he was passing eight or nine stools a day and there was much mucus and blood. He had lost two stones in weight since the onset of his illness. He looked thin and had a pale and sallow complexion. I took him into St. Mark's Hospital and examined him with the sigmoidoscope under an anæsthetic. The whole of the mucous membrane of the rectum and of the lower part of the sigmoid flexure was rough and very coarsely granular, suggesting the appearance seen in granular pharyngitis. There was extensive ulceration of the mucosa, 
Table of Cases of Colitis.

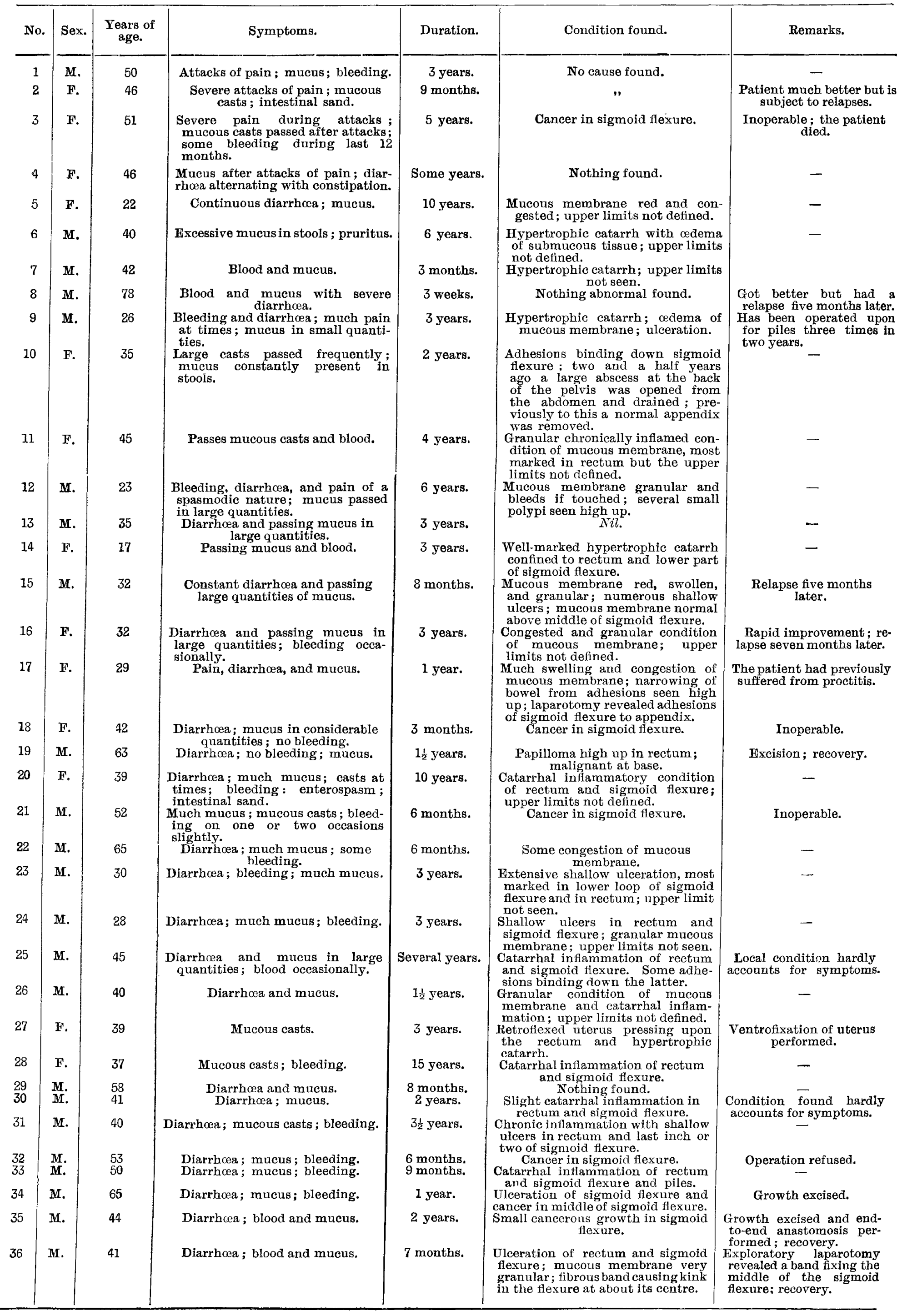


the ulcers being irregular in outline and shallow, involving only the superficial layers of the mucosa. Above the centre of the sigmoid flexure the ulcers were much less numerous. On digital examination some enlarged glands could be felt in the meso-rectum and abdominal palpation revealed a distinct thickening in the left iliac fossa. He was treated by large oil injections into the colon and irrigation with potassium permanganate for a fortnight. Under this treatment the ulcers healed and all the symptoms cleared up. But as there was still a distinct thickening in the left iliac fossa and as I did not feel certain that there might not be a growth higher up in the sigmoid flexure than we were able to see with the sigmoidoscope I thought it advisable to perform exploratory laparotomy. On opening the abdomen I found there was no growth but the walls of the sigmoid flexure were very much thickened, so much so that the bowel in places felt like a thick indiarubber tube. There were also numerous enlarged glands in the meso-sigmoid. In the centre of the sigmoid Hexure there was a thick fibrous band about three-quarters of an inch broad binding down the sigmoid flexure into the left iliac fossa and cansing a kink in the bowel. The transverse colon was normal, as were also the crcum and appendix. All the other organs were normal. I divided the fibrous band and closed the abdomen. After he had got over the operation the previous treatment was continued with the addition that he was given von Noorden's diet, and he was discharged apparently quite well at the beginning of April. With the sigmoidoscope it could be seen that all the vlcers had healed and although the surface of the mucosa was still rough and granular it was decidedly better than at the first examination.

In four cases there was a chronic hypertrophic catarrh associated with cedema of the submucous tissue. On examining such a case with the sigmoidoscope the mucosa is seen to be much paler than normal and to be thrown into numerous folds or concentric rings. The mucous membrane bulges forward into the bowel lumen, giving the appearance of a partial intussusception at the point examined. The mucous membrane is thickened and oedematous, though its surface retains its normal shiny appearance. The sigmoidoscopic appearances are very characteristic and cannot be mistaken. This condition was associated in all my cases with excessive secretion of gelatinous mucus.

Severe pruritus was present in two of the cases and the patient in Case 6 consulted me on this account. This patient got better after regular irrigation of the bowel with an alkaline lotion and the use of astringent injection of krameria, and his pruritus, which had existed for many years, was cured. I have seen this hypertrophic catarrhal condition of the mucosa in two cases of pruritus in which the patient did not give a history of colitis.

Cancer as a cause of colitis.-In seven (Cases 3, 18, 19, 21, 32,34 , and 35 ) out of my series of 36 cases the colitis was due to a tumour within the bowel. In all of these cases there was considerable doubt about the diagnosis previously to the discovery of the tumour and several of the patients had previously been under treatment for colitis. In looking through my cases of cancer of the rectum I find there are several in which the symptoms were those of colitis rather than cancer, but $I$ have not included them as there was at no time any doubt about the diagnosis as a digital examination revealed the true state of affairs.

It is impossible from the symptoms alone to distinguish those cases of colitis which are due to cancer from the other forms. The presence of blood in the stools is of no value. In Cases 18 and 19 there was no blood and only a very small amount of blood on two occasions in Case 21. Definite mucous casts may be passed in cases of cancer, as is shown by Cases 3 and 21. In both these cases typical mucous casts had been passed on several occasions. The age of the patient does not help us much, as in Case 18 the patient was only 42 years of age, and only two of the others were over 55 years, while the average age for all the cases is 43 years. The time during which the symptoms have existed will not exclude cancer, as in Case 19 symptoms had existed for one and a half years and in Case 3 for five years.

Case 3 is a remarkable one. The patient had had symptoms of a severe colitis for five years; she had passed mucous casts of the bowel after acute attacks of abdominal pain. She had laparotomy performed twice. What was found on the first occasion $I$ do not know but at the second operation an adherent ovarian cyst was removed. When I saw her she had a malignant growth at the upper end of the sigmoid flexure. A short-circuiting operation was subsequently performed by another surgeon but she died from intestinal obstraction shortly afterwards.

In Case 18 a large growth in the sigmoid flexure was discovered on examining the patient with the sigmoidoscope. I subsequently performed colotomy after finding that the growth could not be removed. In Case 19 I removed the growth, which was a large papilloma becoming malignant at its base, and the patient has remained well since, a period of over two years. In Case 21 the growth was inoperable. In Case 32 the growth was seen high up in the sigmoid flexure through the sigmoidoscope. The patient unfortunately refused operation. In Case 34 the growth was situated 22 centimetres from the anus. It was seen through the sigmoidoscope and could not have been diagnosed otherwise. This patient had numerous small ulcers scattered over the mucous membrane of the rectum and sigmoid flexure below the growth. Mr. Mayo Robson subsequently excised the growth from the abdomen and anastomosed the ends of the bowel.

Case 35 is of particular interest. The patient was an exceedingly neurotic man, aged 46 years, who was sent to me by Dr. G. Baynton Forge. For nearly two years he had been under treatment by different practitioners for ulcerative colitis and during that time he had been passing blood and mucus in his stools almost continuously. There were no constipation and no difficulty in keeping the bowels open at any time. He had been putting on weight slowly for about two months before I saw him. On examining him with the sigmoidoscope I found a small malignant growth, of about the size of a shilling, growing from the anterior wall of the sigmoid flexure about six inches above the upper end of the rectum. The mucous membrane above the growth was inflamed and resembled the condition seen in non-malignant cases of colitis. I excised the growth and a V-shaped portion of the adjacent mesentery from the abdomen and joined the ends of the bowel end to end by simple suture; the abdomen was closed without drainage. He made a good recovery and returned home in good health about one month after operation. A microscopical examination of the growth showed that it was an ordinary medullary carcinoma.

Among the rarer causes of chronic colitis which have been recorded mention must be made of actinomycosis of the colon, two cases of which are recorded by Beck. Another rare local condition is tuberculosis, of which seven cases are recorded by Beck.

Multiple adenomata of the large intestine.-This is a very curious condition and only a comparatively small number of cases have been recorded. These adenomata are present in great numbers ; in some cases the entire mucosa of the large bowel is covered over with them. In some of the cases only a portion of the bowel is affected, the commonest situations being the sigmoid flexure and rectum. The symptoms are those of a severe colitis. There is often much blood in the stools, but in some of the cases there has been none. One of the most remarkable cases of this disease was that reported by Lilienthal; the whole colon was affected and the patient was ultimately cured by excision of the entire large intestine. A case was reported recently by Dr. Mabyn Read, and the specimen of a case was shown at the Leicester meeting of the British Medical Association in 1905 by Mr. $\mathbf{E}$. Stanmore Bishop. Dr. Carey P. Coombs has also recently reported a case.

Causes of colitis outside the bonel.-Colitis may result from adhesions due to an acute pericolic inflammation. Cases of acute pericolitis are not uncommon and several wellauthenticated cases have been described, notably by Dr. H. D. Rolleston. In one of my cases the patient had an acute attack of abdominal inflammation and a large abscess in connexion with the sigmoid flexure and rectum was found and opened through an abdominal incision. Shortly afterwards she commenced to suffer from well-marked symptoms of mucous colitis, which increased up to the time I saw her three years after the operation. Everything pointed to the adhesions around the sigmoid flexure and binding it down into the pelvis as the cause of her symptoms.

Uterine displacement may be a cause of colitis by interfering with the action of the sigmoid flexure and the evacuation of its contents into the rectum. A retroflexed uterus acts like a ball valve in blocking up the posterior pelvic outlet and causes a very considerable degree of obstruction in some cases. In Case 27, in which laparotomy was performed for colitis, the retroflexed uterus could be seen to block the posterior pelvic outlet almost completely and the 
sigmoid flexure above the point of constriction by the uterus was greatly distended and hypertrophied. Ventrofixation of the uterus resulted in considerable improvement in this patient's condition.

Beck out of 500 cases of chronic colitis found chronic inflammatory lesions in the neighbourhood of the colon in 394.

Coli'is and appendicitis.-It is difficult to say exactly what is the association between appendicitis and colitis. The most usually accepted view is that colitis may result from a chronically inflamed appendix. Dr. H. A. Caley has expressed the view that appendicitis of ten results from colitis. The probability would seem to be that both appendicitis and colitis result from the same causes-namely, incorrect dieting and constipation. That appendicitis can be the cause of colitis is, I think, unquestionable. The evidence in favour of this view is very strong. A certain degree of colitis is usually present in all cases of acute appendicitis, though no definite symptoms of the conditions may show themselves.

In exactly what way appendicitis causes colitis we do not know, but it seems probable that it may produce it in several ways. 1. Appendicitis is generally accompanied by inflam. mation of the ascending colon and cæcum, and in some cases this inflammation of the colic mucosa may spread into the transverse colon and even reach the sigmoid flexure. As Mr. C. B. Lockwood has pointed out, it is not uncommon during the operation for appendicitis to find that the inflammation has spread into the ascending colon. 2. Appendicitis may also cause colitis by the formation of adhesions between the appendix or cæcum and the sigmoid flexure. These adhesions by interfering with the lumen of the gut either through direct constriction or oy causing abnormal flexures and angles may result in a local inflammatory process being set up and the symptoms of colitis subsequently develop. The first result of such adhesions is usually constipation to be followed in a shorter or longer time by diarrhœi and the passage of mucus. This had occurred in Case 17. On opening the abdomen $I$ found the appendix adherent at its tip to the sigmoid flexure and several adhesions binding down the sigmoid flexure and preventing it from rising out of the pelvis. Considerable improvement followed removal of the appendix and sepzration of the adhesions. 3. It would seem probable that a chronically inflamed appendix may cause symptoms of colitis by the frequent discharge of septic material into the bowel. It has become customary to describe the colon and rectum as septic channels, though there is no justification for this. As a matter of fact, septic organisms are not at all usually numerous in the large intestine and the pathogenic flora of the mouth is far more numerous than that of the rectum and colon. Even the skin is a more common habitat for septic organisms than the colon. It is not surprising therefore that a chronically inflamed appendix which is continually discharging septic organisms into the bowel may be the cause of colitis, just as we know that carious teeth and septic conditions of the mouth can cause gastritis.

We have very positive evidence that removal of an inflamed appendix will in many cases lead to a complete cure of colitis. In Mr. Lockwood's paper already referred to he records three such cases where the symptoms of colitis cleared up entirely after the removal of an inflamed appendix. Dr. Hale White, in his valuable paper on membranous colitis, records four cases in which the appendix was removed for symptoms of colitis. In only one of these, however, did a cure of the colitis follow the operation. It is noteworthy, however, that the successful case is also the only one of which it is recorded that the appendix was found to be disensed. Beck found a chronically inflamed appendix to be the cause of colitis in a considerable number of cases of chronic colitis and believes it to be a common cause of colitis. Tuttle records five cases of chronic colitis cured by the removal of an inflamed and adherent appendix. Olmsted of Ontario records a case of severe mucous colitis in which rapid improviment followed the removal of a congested appendix, and Amatt of Ontario records two similar cases.

Adhesions of the bowel as a cause of colitis. - There is every rason to believe that adhesions binding down the large bowel and interfering with its normal movements are an important factor in the causation of colitis. In a great many cases of severe and intractable colitis for which an exploratory Iaparotomy has been performed adhesions binding down the sigmoid flexure, and more rarely the crecum or splenic Hexure, have been found, and their division has been followed By a cure of the condition, though not infrequently the adhesions have re-formed at a later date with a recurrence of the old symptoms. These adhesions have been supposed to arise from long-continued constipation, but it is difficult to understand how adhesions can arise from constipation alone and it seems much more probable that the presence within the bowel of hard retained fæcal masses has set up a local inflammation or definite ulceration and that the adhesions to neighbouring parts have resulted from the local peritonitis thus caused. This seems all the more likely, as adhesions are usually found in those portions of the bowel, such as the sigmoid flexure, ascending colon, and cæcum, where retention of fæcal material most easily occurs. I believe local simple ulceration of the mucosa of the large bowel is much commoner than is generally supposed. I have often observed simple ulcers in the sigmoid flexure with the sigmoidoscope in cases of chronic constipation, though no symptoms of ulceration were present.

The adhesions interfere with the normal peristaltic movements of the colon and a chronic catarrhal inflammation of the mucosa is set up at the points where adhesions have formed.

A floating kidney has been supposed to cause colitis and Tuttle records two cases apparently cured by fixation of an exceptionally mobile kidney. In those cases of colitis associated with a moveable kidney which have come under my observation I have seen no reason to suppose that the condition of the kidney was in any way the cause of the colitis.

It is interesting to observe that symptoms of colitis may occur in cases of abdominal aneurysm. In such cases the colitis apparently results from the pressure of the aneurysm upon some contiguous portion of the large bowel. Tuttle records six cases of abdominal aneurysm in which there was well-marked and intractable mucous colitis.

In six of my cases no local cause for the colitis could be discovered and in two cases (Cases 25 and 30) the local lesion did not seem sufficient to account for the symptoms. In Case 29 in which no local lesion was found the subsequent history of the patient pointed to the presence of a malignant growth high up in the colon, but $I$ have since heard that he is still alive, though not any better, one and a half years after he was examined. In Case 25 it seemed probable that the chief lesion was higher up in the sigmoid flexure than we were able to see. There are thus only six cases out of 36 in which there was no sign of any local lesion. And when we consider the very large part of the colon which cannot be examined it seems at least probable that there was a definite lesion in that part which could not be examined.

Treatment. - The most important factor in the treatment of colitis is undoubtedly to ascertain first of all the real cause of the condition. And the reason the treatment of colitis has often in the past been unsatisfactory is undoubtedly to be assigned to inaccurate diagnosis and treating as a disease what is only a symptom. In the inflammatory conditions rest in bed is advisable at the commencement of treatment. This is more particularly of value in enabling the patient to be kept under close observation. Irrigation of the bowel seems to be the best method of treatment if carried out properly and the inflammatory condition, as a rule, quickly subsides. A skilled nurse who understands how to get the irrigating fluid right up into the colon is essential. The irrigation must be done with a douche and not with a syringe. The reservoir should never be raised more than a foot or two feet above the anus and the fluid must be admitted slowly, with the patient in the semi-prone position. If the condition is at all acute it is better not to begin with irritant in. jections; an alkaline lotion, such as glycerine thymol co. 15 per cent., or sodium bicarbonate one drachm to the pint is very good, or if there is much alceration bydrogen peroxide. Later argyrol 1 per cent., or potissium permanganate 1 in 2000 , is very useful. Large injections of olive oil carefully administered and retained for as long as possible I have found most useful in some of the ulcerated cases. Irrigations are, however, quite useless unless care is taken to see that they reach the affected area, by no means always an easy matter. In some cases where there is local ulceration the ulcers can be painted with nitrate of silver or ichthyol through the sigmoidoscope. Dieting is also very important and the great thing is to get the bowels to act normally. Von Noorden's diet seems the best in most cases.

The most difficult cases to treat would seem to be those in which there is hypertrophic catarrh. They are easily made better but not easily cured. Stimulating injestions are of 
the most value in these cases and treatment must be continued for some time. In the malignant cases operation is, of course, indicated where possible. In those cases where colitis is associated with a thickened and tender appendix, removal of the appendix, so as to get rid of a source of infection, is certainly indicated. In some of the cases of severe ulcerative colitis giving rest to the bowel by establishing a temporary artificial anus may be necessary, but I do not think this will often be the case if the local treatment of the colon by irrigations and applications through the sigmoidoscope is carefully carried out. Before a left inguinal colotomy is performed for colitis a careful examination with the sigmoidoscope should always be made to ascertain whether it is possible to open the bowel above the diseased area. The only advantage of appendicostomy seems to be that it enables the patient readily to perform irrigation of the colon for himself.

In those cases of colitis in which no local cause can be found to account for the symptoms and in which no material improvement in the symptoms has followed a careful and thorough course of medical treatment, I think surgical interference is indicated. Laparotomy should be performed and a thorough examination made of the large intestine; any adhesions which may be found binding down the bowel should be divided; if the sigmoid mesentery is unduly lax, allowing partial volvulus to occur, it should be shortened by stitching. If there is a retroflexed nterus it should be fixed in the normal position and if any thickening or ulceration of the bowel wall can be detected the advisability of resecting that portion of the bowel or of shortcircuiting it must be considered.

I do not think that the operation of ileo-sigmoidostom is advisable except in a few exceptional cases. When very extensive adhesions or ulcerations in the large bowel are discovered, which it does not seem probable can be permanently remedied, this operation should be considered, as it may be the only means of saving the patient from becoming a chronic invalid. It is not an operation, however, to be lightly performed, as the large intestine is a useful and necessary portion of the human body and not, as some people suppose, a useless cesspool, to be cut out or short-circuited without damage to its owner.

I am well aware that it may be said of my cases and observations that owing to the fact that I am a surgeon those cases of colitis in which a local cause is demonstrable tend to preponderate unduly. I admit that this is so, but it may equally well be said that the reverse occurs with those cases coming under the care of the physician. A very large number of cases with symptoms of colitis present themselves for treatment at St. Mark's Hospital and we tbus have exceptional opportunities of observing such cases at that institution.

Bibliography.-Arnott: Brit. Med. Jour., vol. ii., 1906, p. 1279. Beck : Archiv für Klinische Chirurgie, vol, lxxiv., No. 1. Caley : Brit. Med. Jour., June 9th, 1906. Jockwood: Brit. Med. Jour., March 4th, 1905. Nothnagel : Diseases of the Intestines and Peritoneum, edited by $\mathbf{H}$. D. Rolleston, 1904, p. 234. Olmsted : Brit. Med. Jour., vol. ii., 1906, p. 1277 D'Arcy Power : Brit. Med. Jour., Nov. 3rd, 1906. Mabyn Read: TH LaAfCET, March 19th, 1904, p. 797. Rolleston : THE LanCET, April 1st, 1905 p. 854. Tuttle: Diseases of the Anus, Rectum, and Pelvic Colon, secon edition, p. 170. Hale White : THE LANCET, Oct. 28th, 1905, p. 1229.

Cavendish-place, $\mathrm{W}$.

\section{SACRO-COCCYGEAL TERATOID TUMOUR WITH FORMATION OF METASTASES IN THE GROIN ${ }^{1}$}

BY SETON PRINGLE, M.B. DUB,, F.R.C.S. IREL., SURGEON TO MEROER'S HOSPITAL, DUBLIN.

As tumours of the sacro-coccygeal region may be classed among the surgical and pathological curiosities I considered the following case worthy of record, more especially as it presented some unusual features.

The patient, aged 57 years, was admitted to Mercer's Hospital, Dublin, on Sept. 23rd, 1904, having been sent up from the country to have a tumour removed which, as he said, "prevented him sitting down." His history was briefly as follows: 35 years previously he first noticed a small lump of about the size of a nut in the middle line some distance behind the anus. This tumour grew

1 A paper read before the Pathological Section of the Royal Academ of Medicine in Ireland on March 15th, 1907. very slowly and remained quite painless until about four years ago, at that time being of the size of an orange, when it assumed more rapid growth, and he felt what he called "rheumatic pains" in it. Since then growth had proceeded in rather a peculiar manner ; one part would, he said, grow rapidly, forming a painful nodule with the skin over it reddened, but when the nodule reached the size of a hen's egg growth would cease and the skin become pale again; then another part would take on growth and the process repeat itself. For the last few months the upper extremity had been growing rapidly in this manner. At no period did the tremour break down or ulcerate and it was freely moveable for years but had become more fixed in the last 12 months. Some 33 years ago, or two years after the appearance of the above-mentioned tumour, he noticed a small hard lump in his right groin which had continued to grow slowly and quite painlessly up to the time under notice when it was of about the size of a duck's egg. About 20 years ago he discovered another lump also in the right groin above and outside the earlier one and this was about the same size as its neighbour. For the last year he had noticed increasing difficulty in micturition and defæcation, the stools becoming smaller until they were about as thick as his index finger. He said that latterly he had suffered from "rheumatic pains" in his right hip and down the thigh to the knee but that otherwise he was quite strong, had not lost much weight, and his chief complaints were that he was unable to sit for any length of time and that pressure or cold caused pain in the lump. He knew of no relatives who had suffered from similar tumours, and his own children, the youngest being 20 years old, showed no abnormality.

On examination a large tumour was found over the sacral region, the general character of which can be well seen in the accompanying illustration (Fig. 1.) It measured 15 inches

FIG. 1.

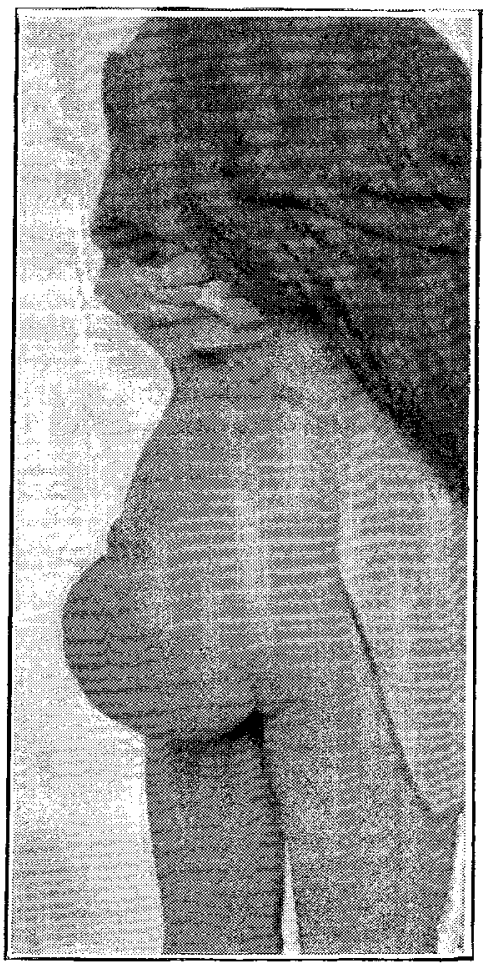

The sacro-coccy-geal teratoma betore removal.

over all from the upper to the lower extremity and 11 inches from side to side, the base extending from the upper border of the sacrum to the anal margin and from slightly outside the left margin of the sacrum to well on to the right gluteal region. Its surface showed irregular lobulations; the skin over most of the mass was mottled blue but over the upper pole it was red and inflamed. There was no induration round the base and the adjacent skin appeared perfectly normal. On palpation the tumour was found to be more or less fixed apparently to the back of the sacrum, but it could be moved to a certain extent from side to side. The skin covering it could not be moved apart from the tumour, but this appeared to be due to its tightly stretched condition. The consistence of the growth was not uniform, most parts being 